\title{
Editorial
}

\section{Articular cartilage restoration: thinking vertically and horizontally}

If we explode the terms "cartilage" and "regeneration" in PubMed we get 8,196 results (search date, 31 May 2016). If we do the same with a search engine like Google, we get around 560,000 results. Instead, a search strategy based on explosion of the terms "cartilage" and "repair" gives us 11,147 and 825,000 results using PubMed and Google, respectively.

Manuscripts reporting clinical or pre-clinical studies on the use, validation or efficacy assessment of new therapeutic strategies for the treatment of articular cartilage lesions tend to be, in two sections in particular (the Introduction and the Conclusions), disturbingly similar. Many of these scientific studies start by saying something like: "articular cartilage lesions are a challenging problem, because damaged cartilage does not repair itself" and end with remarks like: "treatment $X$ made it possible to obtain good repair of the chondral lesion; however, the newly formed tissue is not hyaline cartilage".

Why is it that, with all the cartilage treatment solutions that have been proposed and adopted, research continues to tread the same old path and, despite starting from different points and advancing by different means, seems unable to get beyond the fibrocartilage repair tissue, which can be more or less differentiated? Probably what we should be doing is looking behind and between the facts in order to find the "why" rather than the "that"; in other words, rather than looking for another solution "that" treats cartilage, we should instead be trying to understand "why" it is not proving possible to find a definitive solution to the problem.

One of the important aspects characterizing the strategy of scientific research, and determining its results, its timing and the professional experts involved, is the way of approaching the scientific question, in other words the direction of scientific thinking-vertical or horizontal - when addressing a problem.

Vertical thinking is an analytical and sequential process that is widely used by researchers because it arrives at a solution to the problem faster than the horizontal thinking strategy does. Horizontal thinking, unlike vertical thinking, leads to a broad rather than an in-depth investigation.

Howard Gardner has written about two types of "extraordinary minds", which he calls "Masters" and "Makers" (1). Masters are vertical-thinking experts while Makers are horizontal-thinking visionaries. Edward De Bono coined the term "lateral thinking" to define horizontal thinking (2). In general, lateral thinking means being creative and thinking outside the box.

Each of these two strategies has advantages and disadvantages.Vertical thinking focuses primarily on how to solve a given problem. "Vertical thinkers" usually know everything about something. Horizontal thinking usually focuses on why a problem occurs. "Horizontal thinkers" usually know something about everything, related to their field.

The most outstanding research results are those produced by interdisciplinary efforts. Investigating a single field in order to find an answer will solve a problem, however making links between two or more fields in order to find an answer will lead to a break through. This is why horizontal thinking is linked to break through research (3).

This issue of Joints contains some examples of vertical thinking applied to the problem of cartilage restoration: Manunta et al. (4) present the results of an ovine in vivo study on the use of embryonic cells in the treatment of osteochondral defects of the knee. Usuelli et al. (5) report the results of a clinical study on the treatment of osteochondral lesions of the talus using the autologous collagen-induced chondrogenesis technique. Finally, Manunta's working group also analyzes the effects of nanofractures compared to microfractures on subchondral bone remodeling in an ovine in vivo study of the treatment of chondral injuries of the knee (6). These studies address the cartilage problem in three different ways, each following a "vertical" approach of high scientific merit and considerable methodological quality. With regard to the research by Manunta et al. $(4,6)$, however, the multidisciplinary approach and the attempt to verify the result through different paths is the result of a horizontal thinking approach, too, and as such is both commendable and promising. Quite probably, the ability to think outside the box will be the key to understanding what currently escapes us with regard to the cartilage regeneration process - that "essential" something that, as such, is "invisible to the eyes" (7).

References

1. Gardner HE. Extraordinary minds: portraits of 4exceptional individuals and an examination of our own extraordinariness. New York, Basic Books, 1997.

2. De Bono E. Lateral thinking: creativity step by step. New York, Harper \&Row, 1970.

3. https://jaraads.wordpress.com/2009/11/22/thinking-strategies-vertical-vs-horizontal/

4. Manunta AF, Zedde P, Pilicchi S, et al. The use of embryonic cells in the treatment of osteochonadral defects of the knee: an ovine in vivo study. Joints. 2016;4(2):70-79.

5. Usuelli FG, Grassi M,Manzi L, et al. Treatment of osteochondral lesions of the talus with autologous collagen-induced chondrogenesis: clinical and magnetic resonance evalutaion at one-year follow up. Joints. 2016;4(2):80-86.

6. Zedde P, Cudoni S, Giachetti G, et al. Subchondral bone remodeling: comparing nanofracture with microfracture. An ovine in vivo study. Joints. 2016;4(2):87-93.

7. de Saint-Exupéry A. The Little Prince. Ware, Wordsworth Editions, 1995. 